\title{
Effectiveness of 2-methoxyestradiol in alleviating angiogenesis induced by intracranial venous hypertension
}

\author{
Xiang Zou, MD, Liangfu Zhou, MD, Wei Zhu, MD, PhD, Ying Mao, MD, PhD, and \\ Liang Chen, MD, PhD
}

Department of Neurosurgery, Huashan Hospital, Fudan University, Shanghai, China

\begin{abstract}
OBJECTIVE Intracranial dural arteriovenous fistulas (DAVFs) are complex intracranial vascular malformations that can lead to hemorrhage. The authors recently found that chronic local hypoperfusion seems to be the main cause of angiogenesis in the dura mater, which leads to the formation of DAVFs. As a natural derivative of estradiol, 2-methoxyestradiol (2-ME) has an antiangiogenic effect and can be used safely in patients with advanced carcinoid tumors. This study was conducted to examine the antiangiogenic effects of 2-ME on a rat DAVF model.
\end{abstract}

METHODS Male Sprague-Dawley rats $(n=72)$ were used in the experiments. Intracranial venous hypertension was induced for modeling, and 2-ME was used in the early or late stage for treatment. The effects were examined by immunohistochemistry, Western blot analysis, and quantitative real-time polymerase chain reaction assays.

RESULTS 2-Methoxyestradiol significantly reduced angiogenesis in the dura in early- and late-intervention treatment groups, as proven by the results of immunohistochemical staining, Western blotting, real-time polymerase chain reaction assays, and microvessel density counts. The antiangiogenic effect even lasted for up to 2 weeks after 2-ME cessation.

CONCLUSIONS These data collectively suggest that 2-ME can reduce the angiogenic effect caused by venous hypertension in a rat DAVF model, mainly by suppressing the inhibitor of differentiation 1 (ID-1) and hypoxia-inducible factor $1 \alpha(\mathrm{HIF-1} \alpha)$ pathways.

http://thejns.org/doi/abs/10.3171/2015.6.JNS15159

KEY WORDS angiogenesis; DAVF; HIF-1 $\alpha$; ID-1; rat; 2-methoxyestradiol; vascular disorders

I NTRACRANIAL dural arteriovenous fistulas (DAVFs) are fed by dural arteries with drainage into the dural venous sinuses, meningeal veins, or cortical veins and account for $10 \%-15 \%$ of intracranial vascular malformations. ${ }^{15}$ Hemorrhage is a severe complication of DAVFs and is closely related to the progression of the disease to venous ectasia and high-grade Borden-type DAVF.5 Although surgery and endovascular intervention are primary treatments for DAVFs, the complexity of the angioarchitecture often limits the effectiveness of those therapies. Radiotherapy is a new treatment choice that seems to be relatively safe and effective, but outcomes are still related to cortical venous drainage and lesion size. ${ }^{6}$

We recently found that chronic local hypoperfusion seems to be the main cause of angiogenesis in the dura mater, which leads to the formation of DAVFs. ${ }^{1}$ 2-Methoxyestradiol (2-ME) is a natural derivative of estradiol.
Recent oncology studies have shown that 2-ME is a safe antiangiogenic medicine that can be used to reduce tumor vasculature. ${ }^{13}$ Considering the hypoxia-induced angiogenesis-related similarities between DAVFs and tumors, we speculated that 2-ME can also reduce the progression of DAVFs. In this study, we sought to test this hypothesis by using a rat model.

\section{Methods}

All experiments were approved by the Animal Care and Use Committee of Huashan Hospital, Fudan University. To exclude potential hormonal effects on DAVF formation and 2-ME intervention, only male Sprague-Dawley rats were used in the experiments; the rats weighed 350-400 g. All the rats were raised and kept in a specificpathogen-free (SPF) II room.

ABBREVIATIONS DAVF = dural arteriovenous fistula; EJV = external jugular vein; GAPDH = glyceraldehyde 3-phosphate dehydrogenase; $\mathrm{HIF}-1 \alpha=$ hypoxia-inducible factor $1 \alpha$; ID-1 = inhibitor of differentiation 1; MMP = matrix metalloproteinase; MVD = microvessel density; RT-PCR = real-time polymerase chain reaction; VEGF = vascular endothelial growth factor; 2-ME = 2-methoxyestradiol.

SUBMITTED February 2, 2015. ACCEPTED June 3, 2015.

INCLUDE WHEN CITING Published online December 11, 2015; DOI: 10.3171/2015.6.JNS15159. 


\section{Surgical and Intervention Protocols}

All rats underwent the surgical procedure after an intraperitoneal injection of $1.5 \%$ pentobarbital sodium at a dose of $0.2 \mathrm{ml} / 100 \mathrm{~g}$. The anesthetic was supplemented as needed during the procedure. All surgical procedures were performed using standard sterile techniques. DAVF formation was induced by using a modification of the model described by Chen et al. ${ }^{1}$ First, the right anterior facial vein was ligated with $10-0$ nylon sutures. Then, the proximal common carotid artery was anastomosed to the distal external jugular vein (EJV) in an end-to-end manner using 11-0 nylon interrupted sutures, which caused retrograde flow through the transverse sinus. The proximal segment of the EJV and the initial portions of the right external carotid and internal carotid arteries were obliterated separately. Through a second incision made below the left ear, the left terminal end of the transverse sinus was exposed and obliterated with bipolar coagulation. Afterward, a central craniotomy was performed from the coronal to the lambdoid suture and to $2.5 \mathrm{~mm}$ from the midline using an electronic drill. The superior sagittal sinus was exposed and ligated with 10-0 nylon sutures. A sketch of this model is shown in Fig. 1 upper.

The DAVF rats were randomly assigned to 1 of 3 groups based on the intervention protocol (18 rats in the late-intervention group and 24 rats each in the early-intervention and vehicle groups), and each group was then randomly divided into 4 subgroups according to the period between surgery and the time at which they were killed $(4,8,12$, or 16 weeks for the early-intervention and vehicle groups and 8,12 , or 16 weeks for the late-intervention group [6 rats each]). Rats in the early-intervention group received an intraperitoneal injection of 1\% 2-ME (1:100 dimethyl sulfoxide/phosphate-buffered saline) solution at a dose of $0.06 \mathrm{ml} / 100 \mathrm{~g} /$ day starting immediately after surgery and continuing for 2 weeks. ${ }^{2,4}$ Each rat in the late-intervention group received its first injection 4 weeks after surgery, and the injections were continued daily for 2 weeks. In the vehicle group (blank control group), each rat was injected with solvent daily for 6 weeks. A diagram of the intervention protocol is shown in Fig. 1 lower. For the sham operation $(n=6)$, the EJV, common carotid artery, posterior facial vein, left terminal end of the transverse sinus, and superior sagittal sinus were exposed, but no further manipulations were performed. In total, 72 rats were included in the further experiments. For each group, 3 rats were used for immunohistochemical analysis, and 3 rats were used for Western blot analysis and real-time polymerase chain reaction (RT-PCR) analysis. All 6 rats in each group were used for plasma enzyme-linked immunosorbent assay examination.

\section{Immunohistochemistry}

Dural tissue samples were fixed in $10 \%$ formalin, embedded in paraffin, sectioned at $4-\mu \mathrm{m}$ thicknesses, and stained with anti-CD31 (rabbit monoclonal antibody [Abcam, HK Ltd.]), inhibitor of differentiation 1 (ID-1), hypoxia-inducible factor $1 \alpha$ (HIF-1 $\alpha)$, vascular endothelial growth factor (VEGF), and matrix metalloproteinases 2 and/or 9 (MMP-2/9) antibodies (rabbit polyclonal antibodies [Abcam, HK Ltd.]). The secondary antibody was goat anti-rabbit immunoglobulin. Five random areas were selected in a low-power field $(\times 100)$ in 5 sections, and the numbers of VEGF-, MMP-2/9-, HIF-1 $\alpha-$, and ID-1-positive cells were counted in $\times 200$ fields. Microvessels marked by CD31 (lumen diameter $<10$ cells) were measured in 5 random $\times 200$ fields in 5 sections from each dura, and the microvessel density (MVD) was calculated. The immunohistochemical assessment of the microvessels was performed by 2 independent observers who were blinded to group assignments.

\section{Western Blot Analysis}

The total, nuclear, and cytoplasmic proteins $(100 \mu \mathrm{g})$ of the dural tissue were size-fractionated by using sodium dodecyl sulfate-polyacrylamide gel electrophoresis (SDS-PAGE) and transferred to Immobilon-P membranes (Millipore). The blotted membranes were incubated with primary antibodies against ID-1, HIF-1, VEGF, MMP-2/9, and glyceraldehyde 3-phosphate dehydrogenase (GAPDH) (Abcam, HK Ltd.), followed by incubation with a horseradish peroxidase-conjugated secondary antibody (Jackson Laboratories). The immunoreactivity was detected by using an enhanced chemiluminescence reaction system (Amersham Pharmacia Biotech). Plasma VEGF, MMP2/9, and 2-ME levels were also measured by a sandwich enzyme-linked immunosorbent assay (R\&D Systems) according to the manufacturer's protocols.

\section{RT-PCR Analysis}

Total RNA was isolated from the dural tissues using TRIzol reagent (Gibco BRL). The mRNA expression levels of ID-1, HIF-1 $\alpha$, and GAPDH were evaluated by using RT-PCR analysis. The primers were as follows (forward/ reverse): ID-1, GGCTGCTACTCACGCCTCAA/RCA CTGATCTCGCCGTTCAGG; HIF-1 $\alpha$, GGCTGCTACT CACGCCTCAA/CACTGATCTCGCCGTTCAGG; and GAPDH, ACCACAGTCCATGCCATCAC/TCCACCAC CCTGTTGCTGTA. The melting curves and quantifications were analyzed by using LightCycler and Rel Quant software, respectively. All PCRs were performed in at least triplicate. The PCR products were subjected to electrophoresis on $1.5 \%$ agarose gels, scanned, and semiquantitated by using ImageQuant software (Kodak 1D V3.53).

\section{Statistical Analysis}

All data are presented as means $\pm \mathrm{SD}$. The data were analyzed using independent-sample t-tests and 1-way ANOVA. Any $\mathrm{p}$ value of $<0.05$ was considered statistically significant. All statistical analyses were performed using SPSS 16.0 for Windows.

\section{Results}

\section{2-ME Suppressed the Expression of Angiogenic Factors and in the Dura}

We detected highly positive staining of angiogenic factors in the dural tissue of DAVF-model rats in the vehicle group from 4 to 16 weeks. It is notable that $2-\mathrm{ME}$ was 

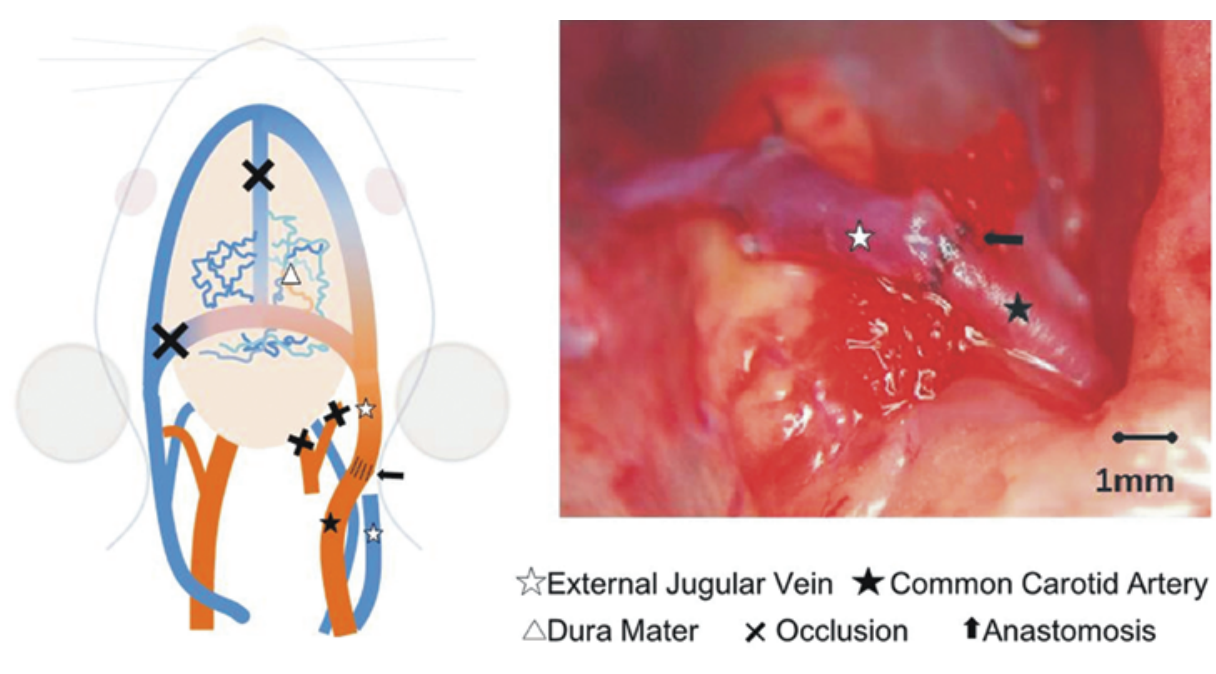

¿̧External Jugular Vein $\star$ Common Carotid Artery

$\triangle$ Dura Mater $\times$ Occlusion Anastomosis

Vehicle

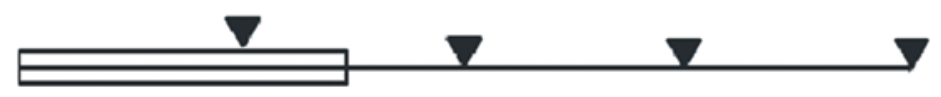

Early Intervention

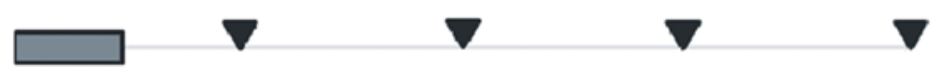

Late Intervention

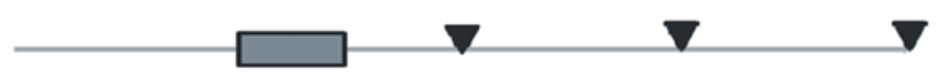

Time Axis

o Week

4 Week

8 Week

12 Week

16 Week

Vehicle

2-ME Intervention

Sacrifice

FIG. 1. Illustrations of the DAVF rat model and intervention program. Upper: Sketch of the operation and an intraoperative photograph. Lower: Intervention program for each group. Figure is available in color online only.

effective in the early and late stages of intervention. Immunohistochemical staining of VEGF was weak in all the dural vessels and surrounding tissue in both the early- and late-intervention groups $\left(150.3 \pm 46.4\right.$ vs $358.2 \pm 66.2 \mathrm{~mm}^{2}$ [p $<0.001]$ and $133.2 \pm 44.5$ vs $482.4 \pm 88.6 \mathrm{~mm}^{2}[\mathrm{p}<$ $0.001]$ ) around the drug-use window and was maintained approximately 2 weeks after the cessation of 2-ME. In the following weeks, both the early- and late-intervention groups were notably highly positive compared with those in the sham-operation group (Fig. 2). Similar results were also found with ID-1, HIF-1 $\alpha$, and MMP-2/9 staining.

\section{2-ME Reduced MVD in the Dura}

The densities of CD31-stained microvessels in the dura were calculated for the different groups and compared. Figure 3A shows that the MVDs continued to increase until the 8th week and then remained stable until 16 weeks in the vehicle-treated group. It is significant that the MVDs were suppressed in both the early-intervention and lateintervention groups. In the early-intervention group (at the 4th week), the MVD was $16.56 \pm 4.80$, approximately half the density in the vehicle group $(35.33 \pm 6.99, \mathrm{p}=0.001)$.
In the late-intervention group (at the 8th week), the MVD was $42.11 \pm 4.70$, approximately $65 \%$ that of the vehicle group $(65.22 \pm 7.38, \mathrm{p}<0.001)$.

\section{Angiogenic Factors Suppressed by 2-ME in the Dura and Plasma}

Western blot analysis was performed for semiquantitative assessment of the angiogenic factors in the dural tissue (Figs. 3 and 4). As expected, the ID-1, HIF-1 $\alpha$, VEGF, and MMP-2/9 protein levels gradually increased in the dura after surgery in the vehicle group over those in the sham-operation group (Figs. 3C and D and 4B-D), and the protein levels became relatively stable after 8 weeks. In general, the angiogenic factors ID-1, HIF-1 $\alpha$, VEGF, and MMP-2/9 were suppressed by 2-ME in the early-intervention group at the 4th week and in the late-intervention group at the 8th week (vehicle-treated group $\gg>$ early- and late-intervention groups $>$ sham-operation group). In the early-intervention group at the 4th week, ID-1, HIF-1 $\alpha$, VEGF, MMP-2, and MMP-9 levels were significantly decreased compared with those in the vehicle group $(2.15 \pm$ 0.03 vs $3.20 \pm 0.36$ [p < 0.001]; $0.58 \pm 0.07$ vs $1.35 \pm 0.01$ 


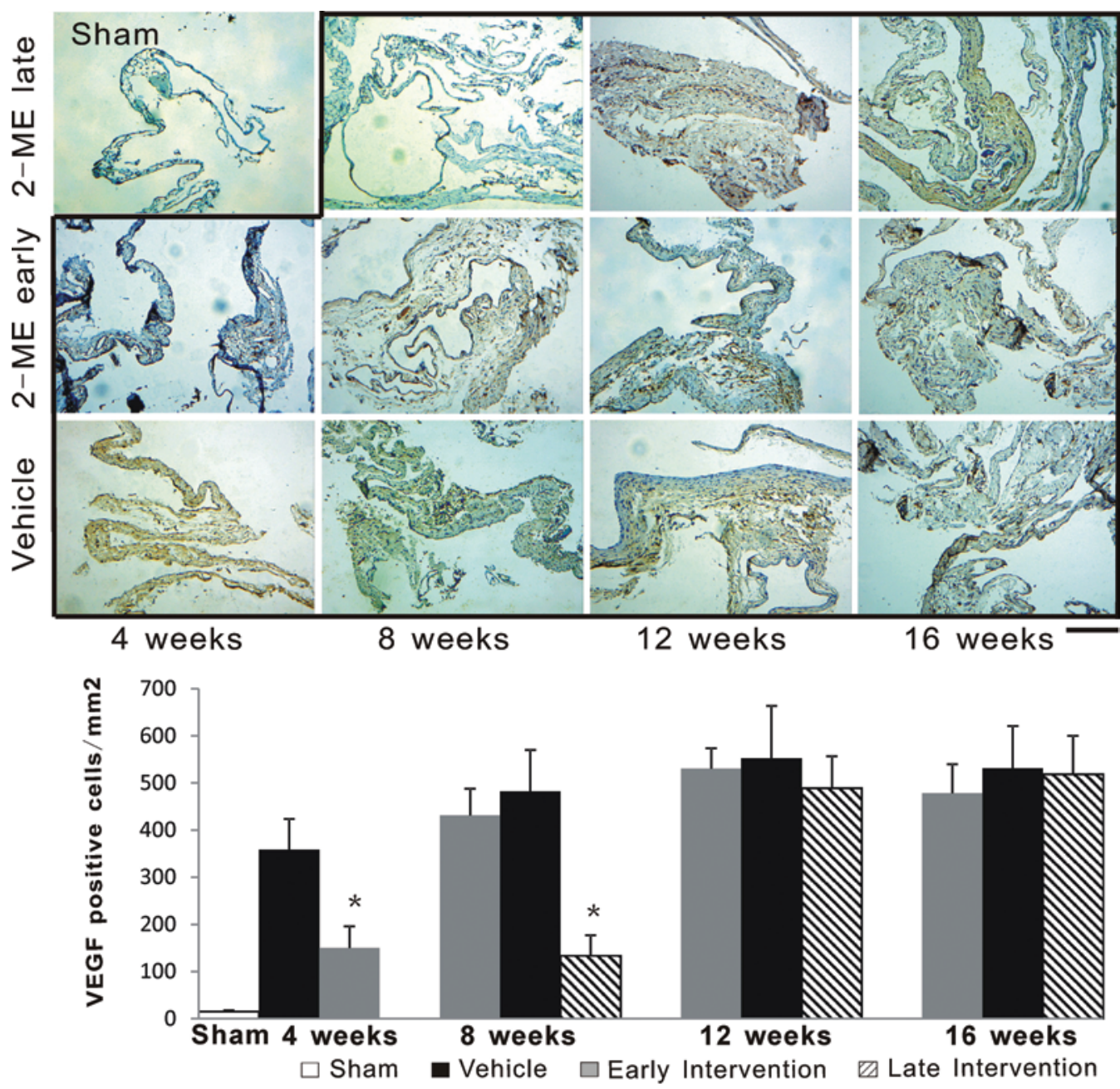

FIG. 2. Upper: VEGF staining of dural sections from each group. Bar $=200 \mu \mathrm{m}$. Lower: VEGF-positive cell counts in each group. ${ }^{*} p<0.05$ versus the vehicle-treated group at the same time points $(n=3)$. Figure is available in color online only.

[p $=0.001] ; 3.38 \pm 0.20$ vs $5.27 \pm 0.31[\mathrm{p}<0.001] ; 1.13 \pm$ 0.17 vs $2.90 \pm 0.48[\mathrm{p}=0.004]$; and $0.97 \pm 0.07$ vs 3.23 \pm 0.36 [p $=0.003]$, respectively). In the late-intervention group at the 8th week, ID-1, HIF-1 $\alpha$, VEGF, MMP-2, and MMP-9 levels decreased to various extents compared with those in the vehicle group $(1.04 \pm 0.08$ vs $7.57 \pm 1.00$ [p < 0.001]; $0.89 \pm 0.01$ vs $4.54 \pm 0.36$ [p $=0.002] ; 5.05 \pm$ 0.22 vs $18.03 \pm 1.51[p<0.001] ; 1.58 \pm 0.19$ vs $5.59 \pm 0.07$ $[\mathrm{p}=0.001] ;$ and $1.77 \pm 0.08$ vs $5.86 \pm 0.36[\mathrm{p}=0.004]$, respectively). For rats in the other intervention groups that were killed at the 12th or 16th week, although 2-ME injections reduced their angiogenic factors to levels comparable to those of the corresponding vehicle-treated groups, the protein levels were still much higher than those in the sham-operation group (vehicle-treated group $>$ early- and late-intervention groups $>>$ sham-operation group). In the plasma, VEGF levels were significantly suppressed in both the early- and late-intervention groups at the 4th, 8th, and 12th weeks after surgery (Fig. 5A). However, MMP2/9 levels in the plasma were not remarkably decreased (Fig. 5B and C). The 2-ME concentrations (all measured before the rats were killed) in the plasma showed no differences among any of the groups (Fig. 5D).

\section{2-ME Suppressed mRNA Levels of Angiogenic Factors and the Time Effects of 2-ME on Venous Hypertension-Induced Angiogenesis}

To analyze the time effects of 2-ME on this DAVF model, we examined the mRNA levels of ID-1 and HIF$1 \alpha$ in the dura (Fig. 6). In the vehicle-treated group, the relative levels of ID-1 and HIF- $1 \alpha$ mRNA continued to increase to their peak values at the 8th week. Then, during the following weeks, ID-1 mRNA levels remained relatively stable, whereas HIF-1 $\alpha$ mRNA levels decreased. Compared with those in the vehicle group, ID-1 and HIF$1 \alpha$ mRNA and protein levels in both the early- and lateintervention groups were decreased significantly after the 2-ME injection. 2-Methoxyestradiol was proven to have an antiangiogenesis effect on mRNA and protein levels during the drug-use window, which lasted up to approximately 2 weeks after drug cessation.

\section{Discussion}

The natural prognosis of DAVF is closely related to the flow rate through the fistula and retrograde flow pattern of the disease, and dural angiogenesis is a major patho- 
A

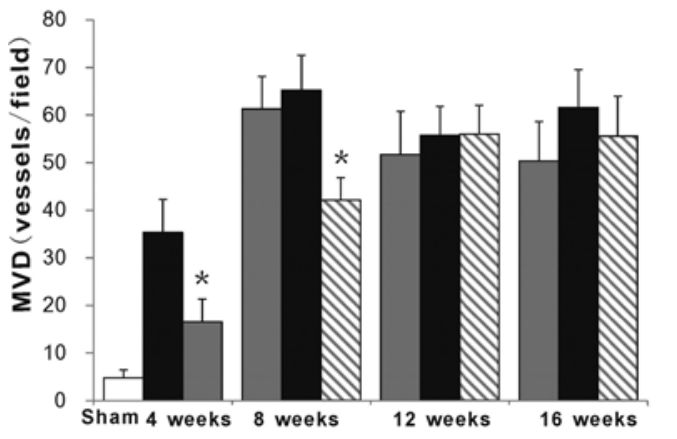

C

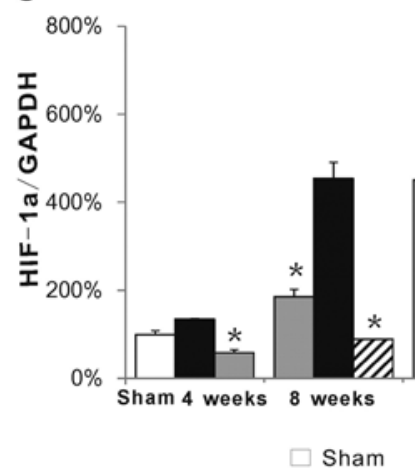

B

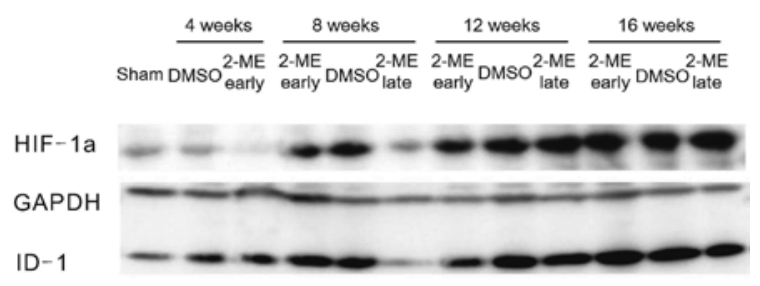

D

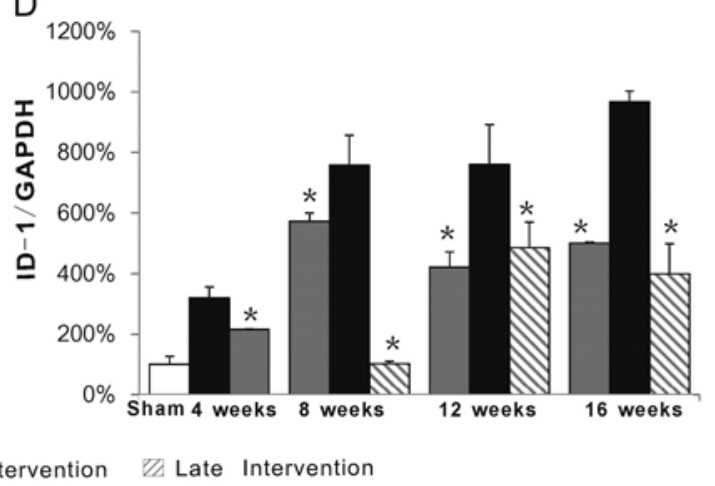

FIG. 3. MVD and Western blot analyses of ID-1 and HIF-1 $\alpha$ in the dura. A: MVD histogram for the different groups. B: Representative bands of ID-1, HIF-1 $\alpha$, and GAPDH. DMSO = dimethyl sulfoxide. $\mathrm{C}$ and D: Histograms showing relative amounts of ID-1 and HIF-1 $\alpha .{ }^{*} p<0.05$ versus the vehicle-treated group at the same time points $(n=3)$.

A

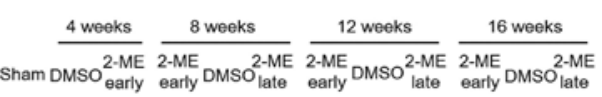

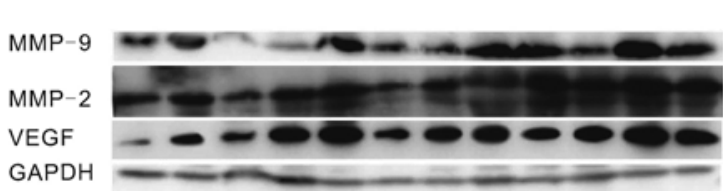

C

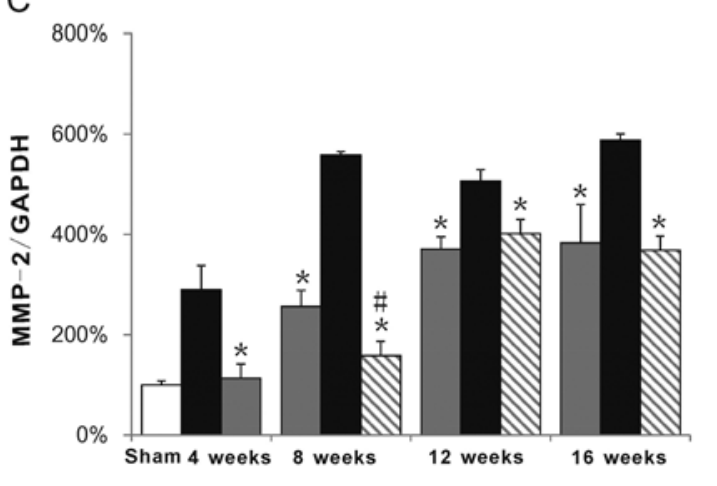

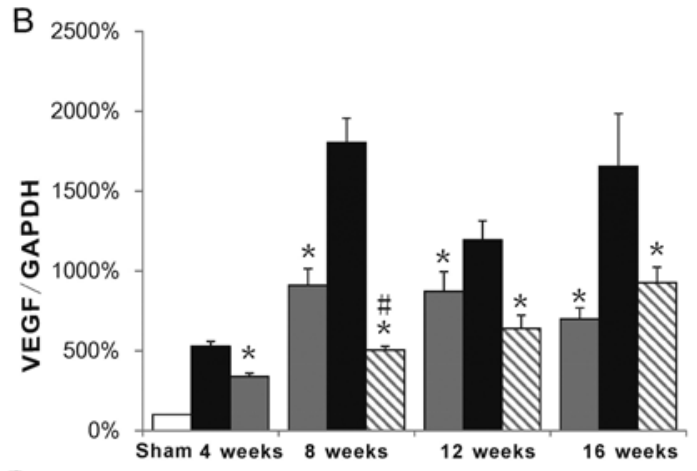

D

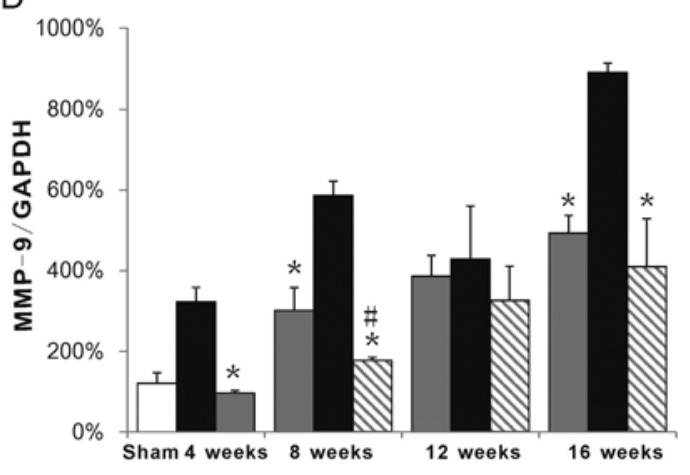

FIG. 4. Western blot analyses of VEGF and MMP-2/9 in the dura. A: Representative bands of VEGF, MMP-2/9, and GAPDH. DMSO = dimethyl sulfoxide. B-D: Histograms showing relative amounts of VEGF and MMP-2/9. ${ }^{*} p<0.05$ versus the vehicletreated group at the same time points $(n=3)$. $\# p<0.05$ versus the early-intervention group at the same time points $(n=3)$. 
A

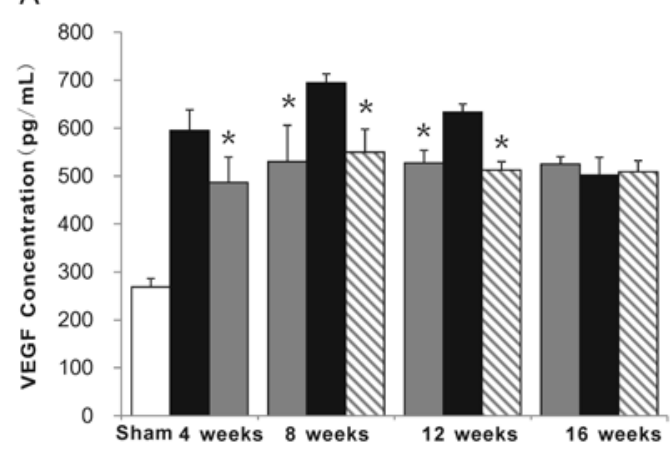

C

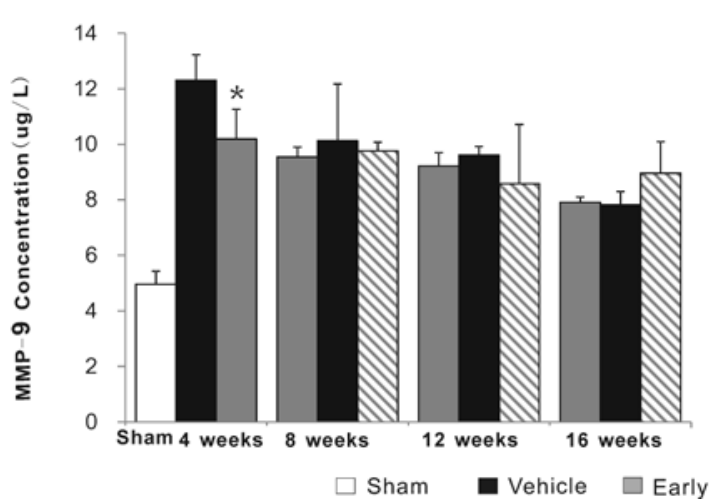

B

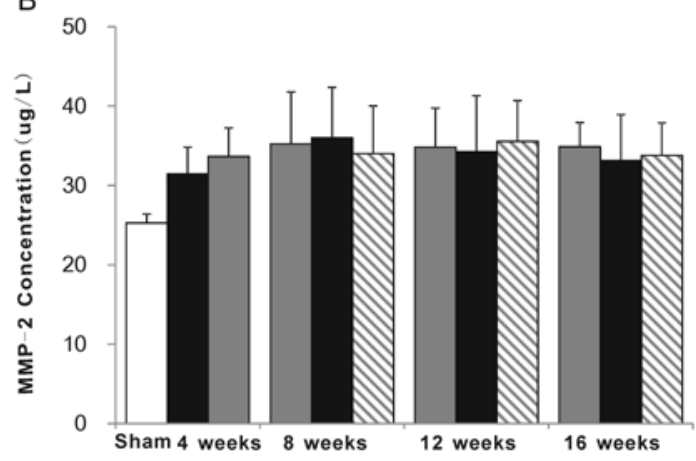

D

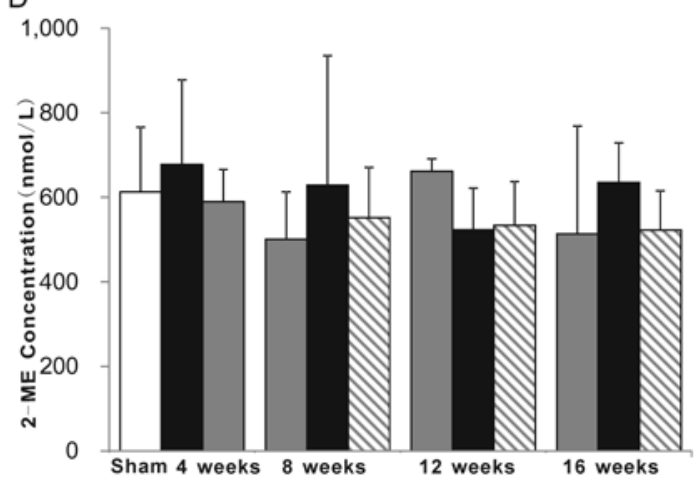

FIG. 5. VEGF, MMP-2/9, and 2-ME levels in plasma (detected by enzyme-linked immunosorbent assays). A: Plasma levels of VEGF, which were significantly suppressed in both the early- and late-intervention groups at the 4th, 8th, and 12th weeks. B: Plasma levels of MMP-2, which were not significantly suppressed by 2-ME in either the early- or late-intervention group at the 4th, 8th, or 12th week. C: Plasma levels of MMP-9, which were suppressed only by 2-ME in the early-intervention group at the 4th week. D: Plasma 2-ME concentrations, which showed no differences among all the groups before the time at which the rats were killed. ${ }^{*} p<0.05$ versus the vehicle-treated group $(n=6)$.

logical change. In 1997, Lawton et al. ${ }^{16}$ first raised the angiogenesis hypothesis for the formation of DAVFs and highlighted venous hypertension, cerebral ischemia, and tissue hypoxia. Zhu et al. ${ }^{21}$ found that the levels of angiogenic factor HIF-1 $\alpha$ and downstream VEGF in the brain could be increased by nonischemic venous hypertension. Our recent study results indicated that venous hypertension might induce chronic regional hypoperfusion and VEGF MMP-9 expression, thus promoting angiogenesis. ${ }^{1}$ A modified rat DAVF model was then created on the basis of these findings. In this study, we confirmed that the angiogenic factors ID-1, HIF-1 $\alpha$, VEGF, and MMP-2/9 and MVD can be induced by venous hypertension and regional hypoperfusion, which might contribute to dural angiogenesis and the initiation of arteriovenous shunting. It is our hope that controlling the angiogenic progress of DAVF lesions provides a new treatment option.

The angiogenesis process is regulated by various angiogenic factors. VEGF and MMPs are key angiogenic factors that can be increased by hypoxia-ischemia, which participate in endothelial cell proliferation and migration.7,20 VEGF and MMPs can be induced by HIF-1 by regulating gene transcription to adapt to tissue hypoxia. ${ }^{11}$ As a member of the ID protein family, ID-1 is believed to regulate cell differentiation, cell-cycle progression, senescence, and apoptosis. ${ }^{18}$ Kim et al. ${ }^{12}$ found that ID-1 can enhance the stability and activity of HIF-1 $\alpha$ by cross-talking in human endothelial cells, thus inducing VEGF expression.

The etiology of DAVF has long been known to relate to sex hormones. A clinical study revealed that serum estradiol levels in patients with DAVF were lower than levels in control patients..$^{14}$ Also, there is a predilection for highergrade DAVFs (according to their Borden type) in males. As a sex hormone, estradiol does not have a direct antiangiogenic effect; however, its metabolite 2-ME is a potent antiangiogenic agent. ${ }^{4}$ Compared with estradiol, this derivative does not exhibit direct estrogenic activity in vitro or in vivo because of its low binding affinity to estrogen receptors. ${ }^{8}$ 2-Methoxyestradiol has been shown to reduce vascular endothelial cell proliferation and migration. Mabjeesh et al. ${ }^{17}$ demonstrated that 2-ME can downregulate HIF-1 $\alpha$ on the protein level and can reduce transcriptional activity in tumors. Huh et al. ${ }^{9}$ confirmed that ID-1 was negatively regulated by $2-\mathrm{ME}$ in tumor and vascular endothelial cells with a dose-effect relationship. Therefore, clinical trials have been approved for patients with cancer. ${ }^{3,19}$ According to a Phase I clinical trial, the maximal 2-ME administration dose is $1000 \mathrm{mg} / \mathrm{day}$, and its half-life is 10 hours in the human body with a daily dose. ${ }^{10}$ Based on previous in vivo studies, ${ }^{2,4}$ 2-ME injection at a dose of $60 \mathrm{mg} / \mathrm{kg}$ per day was considered safe for the rats in this study.

We have shown that 2-ME downregulated ID-1 mRNA 


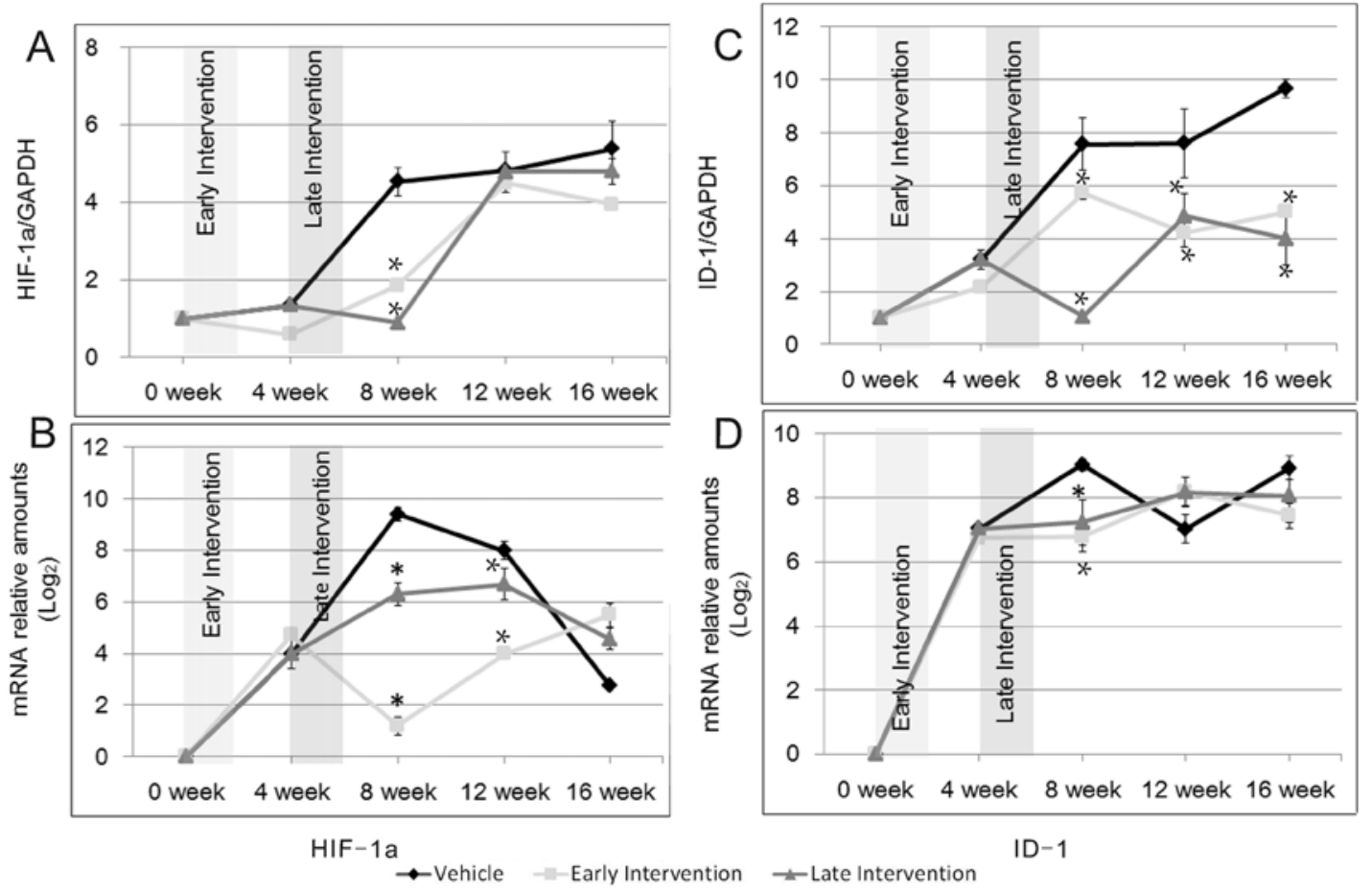

FIG. 6. Time effects of 2-ME on protein and mRNA levels of ID-1 and HIF-1 $\alpha$. The protein and mRNA levels of ID-1 and HIF-1 $\alpha$ were analyzed on time axes. A: HIF-1 $\alpha$ protein levels according to time axis. B: Relative HIF-1 $\alpha$ mRNA levels according to time axis. Relative mRNA amounts were converted to log-base 2. C: ID-1 protein levels according to time axis. D: Relative ID-1 levels according to time axis. ${ }^{*} p<0.05$ versus the vehicle-treated group $(n=3)$.

and protein levels induced by local venous hypertension and regional hypoperfusion. In addition, 2-ME consistently suppressed angiogenic factors in this pathway to various degrees, which led to a decrease of MVD in the dura. Ten weeks after 2-ME cessation, the angiogenic factor levels remained almost half of the levels in the vehicle groups but were much higher than that in the sham group and the MVD could no longer be reduced. That the plasma 2-ME levels at the end-point detection had already decreased to the baseline in the intervention groups implies a persistent requirement for 2-ME administration to control angiogenesis. Because few adverse effects have been identified, long-term administration of 2-ME should be acceptable.

In this study, we could not arbitrarily determine the effectiveness of 2-ME on human DAVF. First, although we tried to replicate the pathogenesis of human DAVF and allowed an assessment of the effect of 2-ME in a rat model, the model we used could only partly represent the features of human DAVF. Second, 2-ME was shown to have a doseeffect relationship with the expression of antiangiogenic factors, and the maximum in vivo dose reached up to 150 $\mathrm{mg} / \mathrm{kg}$. ${ }^{9}$ The 2-ME dose we used in this study was $60 \mathrm{mg} /$ $\mathrm{kg}$ per day and lasted for 2 weeks, so the long-term effect of 2-ME on DAVF formation was not assessed.

\section{Conclusions}

Despite the limitations of this study, our findings collectively imply a potential therapeutic value of 2-ME in this rat DAVF model. Further investigations are needed to determine its long-term effect on DAVF.

\section{Acknowledgments}

This study was funded by project support by the National Science Foundation of China (Grants 81100860 and 81571117).

\section{References}

1. Chen L, Mao Y, Zhou LF: Local chronic hypoperfusion secondary to sinus high pressure seems to be mainly responsible for the formation of intracranial dural arteriovenous fistula. Neurosurgery 64:973-983, 2009

2. Cicek M, Iwaniec UT, Goblirsch MJ, Vrabel A, Ruan M, Clohisy DR, et al: 2-Methoxyestradiol suppresses osteolytic breast cancer tumor progression in vivo. Cancer Res 67:10106-10111, 2007

3. Dahut WL, Lakhani NJ, Gulley JL, Arlen PM, Kohn EC, Kotz H, et al: Phase I clinical trial of oral 2-methoxyestradiol, an antiangiogenic and apoptotic agent, in patients with solid tumors. Cancer Biol Ther 5:22-27, 2006

4. Fotsis T, Zhang Y, Pepper MS, Adlercreutz H, Montesano $R$, Nawroth PP, et al: The endogenous oestrogen metabolite 2-methoxyoestradiol inhibits angiogenesis and suppresses tumour growth. Nature 368:237-239, 1994

5. Gross BA, Du R: The natural history of cerebral dural arteriovenous fistulae. Neurosurgery 71:594-603, 2012

6. Hanakita S, Koga T, Shin M, Shojima M, Igaki H, Saito $\mathrm{N}$ : Role of Gamma Knife surgery in the treatment of intracranial dural arteriovenous fistulas. J Neurosurg 117 Suppl:158-163, 2012

7. Hayashi T, Abe K, Suzuki H, Itoyama Y: Rapid induction of vascular endothelial growth factor gene expression after transient middle cerebral artery occlusion in rats. Stroke 28:2039-2044, 1997

8. Hughes RA, Harris T, Altmann E, McAllister D, Vlahos R, Robertson A, et al: 2-Methoxyestradiol and analogs as novel 
antiproliferative agents: analysis of three-dimensional quantitative structure-activity relationships for DNA synthesis inhibition and estrogen receptor binding. Mol Pharmacol 61:1053-1069, 2002

9. Huh JI, Calvo A, Charles R, Green JE: Distinct tumor stagespecific inhibitory effects of 2-methoxyestradiol in a breast cancer mouse model associated with Id-1 expression. Cancer Res 66:3495-3503, 2006

10. James J, Murry DJ, Treston AM, Storniolo AM, Sledge GW, Sidor C, et al: Phase I safety, pharmacokinetic and pharmacodynamic studies of 2-methoxyestradiol alone or in combination with docetaxel in patients with locally recurrent or metastatic breast cancer. Invest New Drugs 25:41-48, 2007

11. Ke Q, Costa M: Hypoxia-inducible factor-1 (HIF-1). Mol Pharmacol 70:1469-1480, 2006

12. Kim HJ, Chung H, Yoo YG, Kim H, Lee JY, Lee MO, et al: Inhibitor of DNA binding 1 activates vascular endothelial growth factor through enhancing the stability and activity of hypoxia-inducible factor-1 $\alpha$. Mol Cancer Res 5:321-329, 2007

13. Kulke MH, Chan JA, Meyerhardt JA, Zhu AX, Abrams TA, Blaszkowsky LS, et al: A prospective phase II study of 2-methoxyestradiol administered in combination with bevacizumab in patients with metastatic carcinoid tumors. Cancer Chemother Pharmacol 68:293-300, 2011

14. Kurata A, Miyasaka Y, Oka H, Irikura K, Tanaka R, Ohmomo T, et al: Spontaneous carotid cavernous fistulas with special reference to the influence of estradiol decrease. Neurol Res 21:631-639, 1999

15. Kwon BJ, Han MH, Kang HS, Chang KH: MR imaging findings of intracranial dural arteriovenous fistulas: relations with venous drainage patterns. AJNR Am J Neuroradiol 26:2500-2507, 2005

16. Lawton MT, Jacobowitz R, Spetzler RF: Redefined role of angiogenesis in the pathogenesis of dural arteriovenous malformations. J Neurosurg 87:267-274, 1997

17. Mabjeesh NJ, Escuin D, LaVallee TM, Pribluda VS, Swartz GM, Johnson MS, et al: 2ME2 inhibits tumor growth and angiogenesis by disrupting microtubules and dysregulating HIF. Cancer Cell 3:363-375, 2003

18. Ruzinova MB, Benezra R: Id proteins in development, cell cycle and cancer. Trends Cell Biol 13:410-418, 2003

19. Sweeney C, Liu G, Yiannoutsos C, Kolesar J, Horvath D, Staab MJ, et al: A phase II multicenter, randomized, doubleblind, safety trial assessing the pharmacokinetics, pharmacodynamics, and efficacy of oral 2-methoxyestradiol capsules in hormone-refractory prostate cancer. Clin Cancer Res 11:6625-6633, 2005

20. Zhao BQ, Wang S, Kim HY, Storrie H, Rosen BR, Mooney DJ, et al: Role of matrix metalloproteinases in delayed cortical responses after stroke. Nat Med 12:441-445, 2006

21. Zhu Y, Lawton MT, Du R, Shwe Y, Chen Y, Shen F, et al: Expression of hypoxia-inducible factor-1 and vascular endothelial growth factor in response to venous hypertension. Neurosurgery 59:687-696, 2006

\section{Disclosures}

The authors report no conflict of interest concerning the materials or methods used in this study or the findings specified in this paper.

\section{Author Contributions}

Conception and design: Chen, Mao. Acquisition of data: Zou. Analysis and interpretation of data: Zou. Drafting the article: Zou. Critically revising the article: Chen, Mao. Reviewed submitted version of manuscript: Chen, Mao. Statistical analysis: Zou. Administrative/technical/material support: Zhou, Zhu. Study supervision: Chen, Mao.

\section{Correspondence}

Liang Chen, Department of Neurosurgery, Huashan Hospital, Fudan University, Mid Wulumuqi Rd. 12, Shanghai 200040, China.email:dr.chenl@gmail.com. 